\title{
Bibliografien der lokalen Druckproduktion zur Frühen Neuzeit als Quelle der Regionalgeschichte. Neue Möglichkeiten im elektronischen Zeitalter
}

\section{Publikationsformen und Ordnungssysteme}

Bibliografien der lokalen Druckproduktion haben eine lange bibliothekarische Tradition. ${ }^{1}$ Darunter versteht man eine Zusammenstellung aller Werke (auch der Einblattdrucke, Karten, Periodica usw.), die in einer Stadt gedruckt und verlegt wurden, zeitlich auf die Inkunabelzeit und die Frühe Neuzeit bis ca. 1800 begrenzt. Dies erklärt sich aus den politischen Abgrenzungen (Säkularisierung, Mediatisierung, Ende der Freien Reichsstädte). Etwas spätere Zeiteinschnitte lassen sich mit dem Ende der handwerklichen Druckherstellung begründen.

Das umfassende Nachschlagewerk zu den Offizinen des 16. und 17. Jahrhunderts von Christoph Reske unterrichtet jeweils auf den ersten Seiten der jeweiligen Stadt im deutschen Sprachraum darüber, ob eine solche handschriftliche oder gedruckte Auflistung für das 16. und 17. Jahrhundert existiert. ${ }^{2}$ Art und Bedeutung solcher Bibliografien und ihr Nutzen für die Buchforschung sollen wegen der gebotenen Kürze exemplarisch dargestellt und auf den deutschen Sprachraum beschränkt werden. Durch die Impulse, die ihre Herstellung heute durch die nationalbibliografischen Unternehmungen bekommen haben, können wir auf ein wichtiges Arbeitsgebiet unseres Jubilars hinweisen. Dabei haben sich im Laufe der Zeit verschiedene Publikationsweisen und Ordnungssysteme ergeben.

Da sind als ältere Schicht Zettelkataloge und alte handschriftliche Verzeichnisse: $\mathrm{Zu}$ den frühen Beispielen gehört die Zusammenstellung der Kölner

\footnotetext{
1 Vgl. Schmitz, Wolfgang: Ein blinder Fleck. Rheinische Drucke im VD17. Versuch einer ersten statistischen Aussage. In: Bibliothek und Wissenschaft 43 (2010). S. 149-159. Darin habe ich Vorschläge gemacht, wie das VD17 durch lokale Ressourcen systematisch ergänzt und vervollständigt werden könnte. Das hieraus sich ergebende Projekt wird beschrieben von Kötz, Sebastian: Das VD17-Projekt der Universitäts- und Stadtbibliothek Köln. In: ProLibris 19 (2014) H. 4. S. 187-188. Der vorliegende Beitrag knüpft - wenn auch mit anderer inhaltlicher Ausrichtung - daran an. Man möge mir verzeihen, dass ich als Kölner besonders auf die Kölner Verhältnisse als Beispiel zu sprechen komme, die recht ergiebig sind.

2 Vgl. Reske, Christoph: Die Buchdrucker des 16. und 17. Jahrhunderts im deutschen Sprachgebiet. 2. überarbeitete u. erweiterte Aufl. Wiesbaden: Harrassowitz 2015.

ə Open Access. (C) 2018 Wolfgang Schmitz, publiziert von De Gruyter. (G) BY-NC-ND Dieses Werk ist lizenziert unter der Creative Commons Attribution-NonCommercial-NoDerivatives 4.0 Lizenz. 
Drucke durch Ludwig von Büllingen (1771-1848) in seinen Annales typographici Colonienses. In diesem fünfbändigen handschriftlichen Werk verzeichnete der Kölner Kanonikus die ihm bekannt gewordene Druckproduktion aus Köln, grob gegliedert nach Jahrhunderten und darin wieder nach Offizinen. Innerhalb der Offizinen wurden die Titel chronologisch angeordnet. Mancher Titel blieb hier kryptisch, weil die zugrunde liegenden Quellen nicht genau waren. ${ }^{3}$ Obwohl das Verzeichnis heute vielfach überholt ist, erweisen sich einzelne Titel immer noch als nützlich. In den 1980er Jahren erstellte die USB Köln im Rahmen ihrer Mitarbeit aus dem VD16 durch Kärtchenkopien einen Zettelkatalog. Er gliederte das Material nach 1. alphabetischer Reihenfolge der in Köln erschienenen Titel/ Sachtitel, 2. VD16-Bestand in Köln gedruckter Titel geordnet nach Offizinen, darin dann chronologisch. Beide Kataloge verzeichnen die in Köln erschienenen Titel, wo immer sie sich befinden. ${ }^{4}$ Spätere Ergänzungen wurden eingearbeitet, auch eigene Funde. Ähnliche Auflistungen finden wir für einige alte Druckerstädte.

Die Mainzer Stadtbibliothek hat einen so genannten „Mog-Z-Katalog“, der die Druckproduktion für Mainz seit 1454 chronologisch aufführt und dort, wo Lücken im Bestand sind, rosafarbige Karteikarten enthält, manchmal mit Hinweis auf Exemplare in anderen Bibliotheken. Innerhalb eines Jahres sind die Zettel, so gut es geht, nach Druckern/Verlegern alphabetisch sortiert. Ein eigenes D/V-Register gibt es dazu nicht. Leider wird der Katalog nicht mehr à jour gehalten, d. h. es werden keine Karten neu eingelegt, wenn die Stadtbibliothek antiquarisch rückergänzt. ${ }^{5}$ Auch in Nürnberg sind es Zettelkataloge: Aus Kaysers Bücherkunde etc. sind die in Nürnberg seit 1750 gedruckten Bücher herausgezogen und alphabetisch geordnet worden. Nachdem 1945 die Zettelkataloge verbrannt waren, ist bei der Neuverzeichnung der Nürnberger Drucker- und Verlegerkatalog eingerichtet worden: für Nürnberg und Altdorf getrennt nach Offizinen/Verlagen und innerhalb dieser chronologisch für die Druckproduktion $1501 \mathrm{ff} .^{6}$ In Leipzig hatte Dietmar Debes in den 1970er und 1980er Jahren begonnen, eine solche Bibliografie für Leipzig als Zettelkartei aufzubauen, geordnet nach Offizinen und dann chronologisch, die Kartei steht noch im Sonderma-

3 Vgl. dazu Blum, Hans: Ludwig von Büllingen und seine Sammlungen. In: Im Schatten von St. Gereon: Erich Kuphal zum 1. Juli 1960. Köln: Der Löwe 1960. S. 1-12; die Handschrift liegt in fünf Bänden in der USB Köln unter der Signatur 5P 160; Mikrofiche-Ausgabe. Erlangen: Fischer 1997. 48 Mikrofiches: 24x.

4 In einem dritten Katalogteil finden sich Titel aus dem ganzen deutschen Sprachbereich aus dem VD16 im Besitz der USB Köln, sodass ein sehr guter Überblick über die Zusammensetzung des betreffenden Bestands möglich ist.

5 Freundliche Auskunft von Annelen Ottermann (Mainz).

6 Freundliche Auskunft von Christine Sauer (Nürnberg). 
gazin, aber sie ist unvollständig und durch die Recherchemöglichkeiten der VD16-Datenbank völlig überholt. Ebenfalls überholt ist die einzige gedruckte Bibliografie von Helmut Claus. ${ }^{7}$

Es erschienen auch eine ganze Reihe gedruckter Verzeichnisse. Auf der Basis einer schon hoch entwickelten Katalogisierung, gewissermaßen als Übung für den GW, erarbeitete Ernst Voulliéme 1903 ein Verzeichnis des Kölner Buchdrucks für die Inkunabelzeit. Er ordnete nach Autoren und Sachtiteln und lieferte dazu umfängliche Register der Druckwerkstätten, mit chronologisch geordneten Kurztiteln und weiteren druckspezifischen Informationen. ${ }^{8}$ In großem Stil und mehreren Editionsreihen werden solche Kataloge heute durch den Verlag Valentin Koerner in Baden-Baden geliefert. ${ }^{9}$ Hier ist vor allem an seine Reihen des Répertoire bibliographique des livres imprimés en France aux xvie, xviie, xviiie siècle zu erinnern, in dem nach Orten und darin Offizinen gegliedert die Druckproduktion der großen wie der kleinen französischen Orte verzeichnet ist - ein enorm aufwendiges und verdienstvolles Unternehmen. Es gibt bei ihm auch einige Verzeichnisse für deutschsprachige Druckorte.

Bei Verzeichnissen einer lokalen Druckproduktion wird meist ein begrenzter Zeitraum zugrunde gelegt, um die Erarbeitung überschaubar zu machen; Ausnahme sind Druckorte mit kleinerer Produktion. Die Verzeichnisse lassen sich unter Ordnungsprinzipien zusammenfassen, wobei die Zahl der Register unterschiedlich ist:

7 Freundliche Auskunft von Ulrich Johannes Schneider und Thomas Döring (Leipzig). Vgl. Claus, Helmut: Das Leipziger Druckschaffen der Jahre 1518-1539. Kurztitelverzeichnis. Gotha: Forschungsbibliothek 1987. Die Bibliografie ist geordnet nach Offizinen.

8 Vgl. Voulliéme, Ernst: Der Buchdruck Kölns bis zum Ende des fünfzehnten Jahrhunderts. Bonn: Behrendt 1903 (ND Düsseldorf: Droste 1978), die folgenden Zitate S. 3. Nach seinem Vorwort „kann allein eine vollständige Sammlung der Drucke jener Zeit die für eine Geschichte des Kölner Buchdrucks notwendige Grundlage bilden.“ Über die Anlage des Verzeichnisses äußert er sich ebenfalls: „Für die Anordnung der Drucke waren zwei Wege möglich: Die alphabetische nach den Verfassern [...] oder mit Voranstellung des typographischen Gesichtspunktes: Zusammenstellung der Drucke nach den einzelnen Offizinen [...]. Wenn auch zugegeben werden muß, daß diese Methode die wissenschaftlichere ist, so habe ich mich doch mit Rücksicht auf die bequemere Benutzbarkeit des Buches bei Vergleichung und Bestimmung von Inkunabeln zu der alphabetischen Ordnung entschlossen und für die Zusammenstellung der Drucke der einzelnen Offizinen die Tabellenform gewählt, die überdies einen viel leichteren Überblick über den Umfang der Leistungen, die Entwicklung und technischen Fortschritte des einzelnen Druckers gestattet.“

9 Vgl. http://koernerverlag.de/ mit Auflistung der bibliografischen Verlagsaktivitäten. Alle aufgeführten Internetressourcen wurden zuletzt am 3. Dezember 2017 aufgerufen. 
1 alphabetisch nach Autoren/Sachtitel + Register

Diesem Prinzip folgen die Bibliografie der Hamburger Schriften des 16. Jahrhunderts ${ }^{10}$ wie die zum ersten Jahrhundert des Münsteraner Buchdrucks ${ }^{11}$ sowie der Straßburger und der Hagenauer Drucke des 16. Jahrhunderts. ${ }^{12}$

2 nach Druckern, darin chronologisch + Register

Nach Druckern gliedert sich das Verzeichnis der Erfurter Drucke der ersten Hälfte des 16. Jahrhunderts. ${ }^{13}$ Dem gleichen Prinzip folgen Bibliografien der Titel für Ingolstadt, ${ }^{14}$ der Zürcher Drucke des 15. und 16. Jahrhunderts ${ }^{15}$ und für Frankfurt an der Oder im 16. Jahrhundert. ${ }^{16}$ Innerhalb der Jahre stehen die Titel im Alphabet der Autoren/Sachtitel.

3 rein chronologisch + Register

Für Düsseldorf existiert eine die Jahrhunderte umfassende gedruckte Bibliografie in chronologischer Anordnung, innerhalb der Jahre alphabetisch sortiert. $^{17}$

10 Vgl. Kayser, Werner u. Claus Dehn: Bibliographie der Hamburger Drucke des 16. Jahrhunderts. Hamburg: Hauswedell 1968. Das Besondere ist eine exemplarische systematische Übersicht in 14 Gruppen (Sachübersicht).

11 Vgl. Haller, Bertram: Der Buchdruck Münsters 1485 bis 1583. Eine Bibliographie. Münster: Regensberg 1986.

12 Vgl. Benzing, Josef: Bibliographie Strasbourgeoise. Bibliographie des ouvrages imprimés à Strasbourg (Bas-Rhin) au XVIe siècle. Bd. 1-3, Bd. 2 und 3 von Jean Muller. Baden-Baden: Koerner 1981-1986; Benzing, Josef: Bibliographie Haguenovienne. Bibliographie des ouvrages imprimés à Haguenau (Bas-Rhin) au XVIe siècle. Baden-Baden: Koerner 1973.

13 Vgl. Hase, Martin von: Bibliographie der Erfurter Drucke von 1501-1550. 3. Aufl. Nieuwkoop: de Graaf 1968. Etwas Besonderes darin sind Drucke, die fälschlicherweise als Erfurter Drucke aufgelistet worden sind.

14 Vgl. Stalla, Gerhard: Bibliographie der Ingolstädter Drucke des 16. Jahrhunderts. 2. Aufl. Baden-Baden: Koerner 1977; Stalla, Gerhard: Der Ingolstädter Buchdruck von 1601 bis 1620. Die Offizinen Adam Sartorius, Andreas Angermaier und Elisabeth Angermaier. Baden-Baden: Koerner 1980.

15 Vgl. Vischer, Manfred: Bibliographie der Zürcher Druckschriften des 15. und 16. Jahrhunderts. Baden-Baden: Koerner 1991. Drei Register erschließen diese Auflistung: 1. Verfasser, Körperschaften, Anonyma, 2. Mitverfasser, Bearbeiter, Kommentatoren etc., 3. Provenienzen. Für das 15. Jahrhundert werden auch Einblattdrucke einbezogen. Für Zürich (der Bestand von Titeln des 16. Jahrhunderts umfasst ca. 1600 Titel, fast alle im Besitz der ZB Zürich und dort über den AK elektronisch recherchierbar) ist ein elektronischer Druckerkatalog geplant (laut Auskunft Leeu).

16 Vgl. Teitge, Hans Erich: Der Buchdruck des 16. Jahrhunderts in Frankfurt an der Oder. Berlin: Staatsbibliothek 2000, Verzeichnis S. 296-593. Die Erschließungstiefe ist für die erste Hälfte des Jahrhunderts größer als für die zweite. Die Bibliografie ist Teil seiner umfassenden Arbeit über den Frankfurter Buchdruck, die er als Habilitationsschrift vorgelegt hat.

17 Vgl. Verzeichnis Düsseldorfer Drucke (1555-1806). Eine Bibliographie bearbeitet von Manfred Neuber, Marianne Riethmüller u. Rudolf Schmitt-Föller. Wiesbaden: Reichert 2005. http:// 


\section{Titelblätter + Register}

Einen besonderen Weg ging Manfred Kopp 1990 bei den Urseler Drucken: Die Drucke werden in chronologischer Reihenfolge geboten, aber als fotografische Wiedergabe der Titelblätter. ${ }^{18}$

Es gibt jeweils komplementäre Register: Die Gliederung nach Autoren/Sachtiteln erfordert einen Index der Drucker mit zugehörigen Titeln, eine rein chronologische einen der Autoren/Sachtitel und Drucker, eine Ordnung nach Druckern verlangt ein Register der Autoren/Sachtitel. Manchmal gibt es zusätzlich Auflistungen der Beiträger, der Aufbewahrungsorte, Buchhändler und Buchbinder.

Die jeweiligen Vor- und Nachteile wie die Probleme liegen für die einzelnen Ordnungssysteme auf der Hand: Nicht firmierte Drucke müssen nach einem Erschließungsvorgang (Drucker, Jahr) eingeordnet werden, entsprechend gilt das für Titel ohne Verfassernennung. ${ }^{19}$ Wünschenswert ist es natürlich, dass diese Verzeichnisse im Netz frei verfügbar sind. Das ist aber leider nicht immer der Fall. Die meisten Bibliografien verfügen über keine Sacherschließungselemente.

Dennoch gehört die Zukunft auch bei den Druckerkatalogen der elektronischen Datenbank. Für die moderne, zukunftsorientierte Richtung kann Basel als exzellentes Beispiel dienen: Die Basler Universitätsbibliothek erarbeitete für den Basler Buchdruck nach älteren konventionellen Verzeichnissen den Index typographorum editorumque Basiliensium (ITB). Der ITB ist der neue elektronische Basler Buchdrucker- und Verlegerkatalog. Er dokumentiert die Produktion sämtlicher bekannter Drucker und Verleger, die zwischen dem Anfang der Drucktätigkeit um 1468 und dem Jahr 1800 in Basel tätig waren. Er lie-

digital.ub.uni-duesseldorf.de/ihd/content/pageview/4581929. Enthält 862 Drucke, dazu separat 30 Periodica; schließt aus: Einblattdrucke, Kalender, Ablassbriefe, Andachtsbilder und obrigkeitliche Bekanntmachungen (vgl. S. XII, Anm. 3). Gedruckt, aber offen gehalten in der Hoffnung auf weitere Drucke, die aus anderen Bibliotheken etc. gemeldet werden: Register der Personen, Körperschaften und anonymen Titel sowie eines der Drucker, Verleger, Buchbinder und Händler. In ihrem Vorwort weist Irmgard Siebert darauf hin: Mit „der Verzeichnung Düsseldorfer Drucke der frühen Neuzeit wird gar ein ganzes Forschungsfeld zur Druck- und Kulturgeschichte der Stadt eröffnet, das ohne eine institutionelle Vorarbeit wie den vorliegenden Band verschlossen geblieben wäre.“ (o. P.)

18 Vgl. Kopp, Manfred: Die Druckerei zu Ursel 1557-1623. Versuch eines Porträts. Oberursel: Magistrat 1990. Beweggrund ist laut Vorwort unter anderem, dass die Titelblätter der Zeit viel ausführlichere Hinweise geben als bloße normierte Titelaufnahmen. Register der Autoren und Verleger sowie ein Register der Standorte sorgen für die entsprechende Erschließung.

19 Haller, Buchdruck (wie Anm. 11), S. 14 verweist darauf, dass manche Drucke nicht datiert sind und sich der Einordnung entziehen: „Schätzungen des Erscheinungsjahres führen nicht selten zu groben Fehldatierungen.“ 
fert zudem weitere Informationen zu den einzelnen Offizinen. Die Bibliografie befindet sich noch im Aufbau. Aktuell sind alle bis dato bekannten in Basel gedruckten und/oder verlegten Werke des 15. und 16. Jahrhunderts im Katalog verzeichnet. Die Bearbeitung des 17. Jahrhunderts steht kurz vor dem Abschluss, die des 18. wird in den nächsten drei bis vier Jahren folgen. Geplant ist auch, weitere Informationen zu den einzelnen Druckern und Verlegern zu ergänzen (Biografie, Standort der Offizin, Druckermarken, Porträts, Quellen, Sekundärliteratur). Dieser Ausbau wird über einen längeren Zeitraum realisiert. ${ }^{20}$ Elektronische Versionen gewähren erheblich mehr Möglichkeiten der Recherche, z. B. bietet der ITB die Recherche nach Drucken (chronologisch), Offizinen (alphabetisch), Offizinen (chronologisch), Autoren und Stichwortsuche. Positiv ist bei den elektronischen Auflistungen eine mögliche ständige Ergänzung und Verbesserung.

Neben Gesamtübersichten gibt es häufiger solche $\mathrm{zu}$ einzelnen Offizinen. ${ }^{21}$ Vielfach sind sie in Aufsatzform erschienen. In einigen der Vorworte sind die Schwierigkeiten der Titelermittlung im Zeitalter der Zettelkataloge anschaulich beschrieben. Unterschiedlich ist die Wiedergabe der Titel, teilweise streng per Autopsie, jedenfalls soweit möglich, teilweise erfolgt eine möglichst exakte diplomatische Wiedergabe mit Zeilentrennung usw. und in allen möglichen Zwischenformen. Bei den Exemplaren ist meist keine Vollständigkeit angestrebt.

Es ist vornehmlich - wenn wir vom bibliografischen Verlag Valentin Koerner einmal absehen - die Aufgabe von Regionalbibliotheken oder solchen, die auch Regionalaufgaben haben: Stadt-, Universitäts- und Landesbibliotheken. ${ }^{22}$ Es gibt aber noch viele wichtige Druckorte im deutschen Sprachgebiet ohne regionale Bibliografien wie Wien und München.

20 Vgl. http://www.ub.unibas.ch/itb/.

21 Vgl. die Assessorarbeiten aus dem BLI bzw. der daraus hervorgegangenen Abteilung der FH Köln, z. B. Schüling, Joachim: Der Drucker Ludwig von Renchen und seine Offizin. Ein Beitrag zur Geschichte des Kölner Buchdrucks. Wiesbaden: Harrassowitz 1992; Stark, Marcus: Ex officina Melchioris Novesiani. Untersuchungen zur Druckproduktion einer Kölner Werkstatt der Reformationszeit. Wiesbaden: Harrassowitz 2003.

22 Aus dieser Verantwortung bzw. selbst gesetzten Zuständigkeit erwächst auch die Sammlung regionaler Drucke in Neugründungen wie der UB Siegen in ihrer Siegerlandsammlung. 


\section{Der Nutzen eines lokalen Druckerkatalogs}

Die elektronischen Verzeichnisse, besonders die nationalbibliografischen VDUnternehmungen können durch ihre differenzierte Recherchemöglichkeit die Herstellung solcher lokaler Bibliografien enorm erleichtern. ${ }^{23}$ Inwiefern bietet dann eine moderne elektronisch geführte lokale Bibliografie, die jedenfalls im Kern auf den nationalbibliografischen Datenbanken beruht, einen Mehrwert?

Man kann zunächst darauf verweisen, dass auch Personalbibliografien zu einzelnen Dichtern, Gelehrten etc. nicht obsolet geworden sind, obwohl sich die Titel in den übergeordneten Unternehmungen finden. ${ }^{24}$ Es ist neben der intendierten Vollständigkeit eine Frage der Erschließungstiefe. Es gibt eine Vielzahl von Fragestellungen und daraus folgenden Aufgaben.

Das ist zunächst die Übersicht über die lokale Druckproduktion, d. h. über alle Autoren und Werke einschließlich der Einblattdrucke usw., die am Ort gedruckt worden sind, mit Feststellung der Tätigkeitsdauer, gegebenenfalls mit Vorgängern und Nachfolgern (Witwe, Erben usw.). Hier schließt sich eine Übersicht zu der Produktion der einzelnen Verleger/Drucker an, die die Erstellung eines Profils gestattet und Auskunft erteilt über die Zusammenarbeit der Drucker mit Verlegern, eventuell im Rahmen eines Konsortiums. Sie lassen die Bedeutung und Ausrichtung einzelner Vertreter des Buchwesens deutlich hervortreten. Die Daten bilden auch die Grundlage für eine Jahresanalyse, d. h. die Gewichtung der Produktion in zeitlichen Segmenten.

Wichtig ist das Aufspüren fingierter und unfirmierter Drucke aus der jeweiligen Stadt und Zuweisung an eine Offizin, auch solcher, die in den nationalbibliografischen Verzeichnissen noch nicht identifiziert wurden. Hierzu dient eine Sammlung der entsprechenden Typenalphabete, Initialen, Zierleisten, Titeleinfassungen, Holzschnitte, Kupferstiche und Signete. Sie schließt ebenso die entsprechend gekennzeichnete Aufnahme von apokryphen, also bislang nicht identifizierbaren Titeln ein, die später teilweise aufgefunden werden.

Deutlich wird auch, dass manche Drucker in verschiedenen Städten tätig wurden, möglicherweise im Dienste von Verlegern, sodass sich Beziehungen auftun. Interessant sind die Adressangaben der Drucker, denn sie zeigen eventuelle örtliche Veränderungen (Umzüge) in der Stadt auf, die analysiert werden müssen, um Aufschluss etwa über sozialen Auf- oder Abstieg zu erhalten. Schon Reske macht allerdings darauf aufmerksam, dass nicht alle derarti-

23 Eine vergleichbare Analyse solcher Fragen liefert anhand der VD17-Datenbank: Reske, Christoph: Das VD17 als Quelle für die Buchwissenschaft. In: Bibliothek und Wissenschaft 43 (2010). S. 121-138.

24 Vgl. Wilhelmi, Thomas: Sebastian-Brant-Bibliographie. Bern: Lang 1990. 
gen Angaben aus den Titelaufnahmen z. B. des VD17 gewonnen werden können, sondern dass dazu der Blick in die digitalisierten Drucke selbst (Impressum) Hinweise gibt. ${ }^{25}$ Manchmal finden sich Angaben von Bestallungen und Privilegien im Impressum, selbst wenn Archivmaterialien fehlen, ebenfalls bei Drucker- oder/und Verlegersigneten. Ergänzend können dann noch Hinweise auf Leichenpredigten von Druckern/Verlegern mit Angaben von Sterbedatum, Sterbegrund, Beerdigungsdatum, Geburtsdatum, Verwandten und Lebensumständen herangezogen werden. ${ }^{26}$

Notwendig ist eine inhaltliche Erschließung nach Sachgruppen, wie es schon Voulliéme 1903 getan hat. ${ }^{27}$ Das VD17 bietet Gattungsbegriffe. ${ }^{28}$ Wegen des im Verhältnis begrenzten Umfangs kann mit erweiterten Sachgruppen eine verfeinerte Statistik erstellt werden. Die Druckerkataloge vermitteln einen wichtigen Einblick in die geistige Situation einer Stadt zu einer bestimmten Zeit, etwa wenn nonkonformistische Literatur oder religiös unerwünschte Literatur gedruckt wurde. Interessant ist auch eine Statistik nach Sprachen, z. B. hinsichtlich des Anteils des Lateinischen, der mit wissenschaftlichen bzw. theologischen Inhalten zusammengebracht werden kann und zugleich Exportmöglichkeiten eröffnete. Nicht minder interessant sind die Anteile des Griechischen, des Deutschen und der anderen modernen Fremdsprachen, namentlich des Französischen, aber auch Englischen, Italienischen, Spanischen usw. in verschiedenen Literaturgattungen. Archivalische Quellen dienen zur Beantwortung etwa folgender Fragen: Warum sind bestimmte Texte gedruckt worden? Gab es eine Rezeption vor Ort? Dafür wären vor allem Quellen bzw. annotierte Exemplare aus der entsprechenden Region von Interesse. Lokal überliefert ist, wie schon Dünnhaupt vermutete, das ephemere Schrifttum wie Einblattdrucke, Flug- und Streitschriften, Kalender, Praktiken, Liedgut, Universitätsschriften wie Vorlesungsverzeichnisse und Vorlesungen, Dissertationen, Promotionsglückwünsche, Programme und Einladungen, Gelegenheitsschriften zu Taufe, Hochzeit, Tod, Neujahr, Amtseinführung, geistlichen Feiern und Leichenpredig-

25 Vgl. Reske, VD17 (wie Anm. 23), S. 124-125.

26 Reske, VD17 (wie Anm. 23), S. 122 verweist darauf, dass die heutige Druckforschung verstärkt etwas über die Lebenssituation der Drucker erfahren will: Familienmitglieder, Bekannte, finanzielle Lage, Besitz, Verhältnis zur Obrigkeit, Werkstattausstattung, Mitarbeiter, Kollegen, Beteiligung an Druckprojekten und Druckproduktion hinsichtlich Inhalte, Sprache und Zeitraum der Drucktätigkeit.

27 Vgl. bei Voulliéme (wie Anm. 8) die Darstellung des Charakters der ältesten Kölner Literatur, S. LXXIX mit Statistik der Inhaltsgebiete.

28 Vgl. auch die Normdatei der AG Alte Drucke zur Erschließung. 
ten. ${ }^{29}$ Sie fehlen manchmal immer noch in den VD-Datenbanken. Ebenso ist die Einbeziehung im VD16 generell ausgeschlossener Materialien wie Einblattdrucke trotz der großen Zahl lokal eher möglich. Derartige Bestände finden sich in den kleinen Einrichtungen am Ort wie Stadt-, Kirchen- und Archivbibliotheken. In Österreich sind viele kleinere Bibliotheken von Klöstern, Schulen, Adelsfamilien, Pfarrhäusern z. T. nicht bekannt bzw. noch nicht eingespeist. Die Verzeichnung der regionalen Druckproduktion schafft einen wichtigen Einblick in die jeweilige politische, Wirtschafts- und Geistesgeschichte und bietet für entsprechende Studien eine wichtige Basis.

Zur größeren Erschließungstiefe des lokalen Verzeichnisses gehört eine größtmögliche Vollständigkeit des Exemplarnachweises, um Fragen wie Distribution, Preise, Provenienz und Rezeption zu klären..$^{30}$ Daher sollte eine solche lokale Bibliografie möglichst alle erreichbaren Exemplare verzeichnen. Da alte Drucke stets als Individuen zu behandeln sind, auch wenn sie einem Druckvorgang angehören, können sie interessante Abweichungen enthalten und sind daher exemplarspezifisch interessant. Wenn möglich, ist eine Verlinkung mit der Beschreibung von Exemplaren in regionalen Katalogen sinnvoll.

Nützlich sind neben den erwähnten Sammlungen zur Identifikation von Drucken auch Hinweise auf Urkunden, chronikalische Mitteilungen, Bilder von Druckern/Verlegern, Häusern sowie Privilegien und Zensurmitteilungen. Die moderne Buchforschung zielt nicht zuletzt auf den Vergleich mit anderen Druckerstädten, um das Profil der jeweiligen Stadt zu schärfen.

Für die Bibliothek, die sich der Erforschung der lokalen Buchproduktion verpflichtet fühlt, ist ein solcher Druckkatalog für Auskunftsfragen über die lokale Druckproduktion wie für die Steuerung der Erwerbungspolitik hilfreich. Wenn man weiß, was fehlt, kann man in Antiquariats- bzw. Auktionskatalogen zielgerichtet suchen. Bei der Vielzahl der Anforderungen und dem Aufwand ist klar, dass eine solche umfangreich zu nutzende lokale Bibliografie nicht schnell erstellt, sondern nur als „work in progress“ angegangen werden kann.

29 Vgl. Sauer, Christine: Ein Fässchen mit dem Stein der Weisen. Beispiele zur Bedeutung des VD17 als Quelle für lokal- und regionalgeschichtliche Forschungen. In: Bibliothek und Wissenschaft 43 (2010). S. 139-148, hier S. 141.

30 Das VD17 etwa wird zwar nach Auslaufen der DFG-Förderung weitergeführt, aber angestrebt wird vorrangig die Erweiterung durch neue Titelaufnahmen mit einer Bestandsangabe, die Ergänzung weiterer Besitznachweise ist nicht prioritär. Hier können die lokalen Druckerkataloge subsidiär eintreten. Ferner können die von Reske, VD17 (wie Anm. 23), S. 130 angeführten, meist formalen Mängel bei der Übernahme z. B. aus dem VD17 im kleineren Rahmen leichter behoben werden. 


\section{Die Erarbeitung lokaler Druckkataloge}

Die Zusammenstellung der Druckproduktion eines Ortes war vor der elektronischen Verzeichnung in Katalogen sehr schwierig, weil die traditionellen Zettelkataloge, auch Verbundkataloge, keinen Zugriff nach Ort und Verleger erlaubten. Das war immer nur auf dem Umweg über Autor/Titel möglich und bedeutete, dass unbekannte Autoren/Titel so nicht gefunden wurden. Der Umstieg auf elektronische Kataloge hat hier ganz neue Suchmöglichkeiten nach Orten und Druckern/Verlegern eröffnet. Naheliegend ist die Nutzung der großen nationalbibliografischen Unternehmungen. Für die Inkunabelzeit lässt sich der GW entsprechend abfragen. Es sind Verzeichnisse mit einer vorzüglichen Qualität, zuverlässig und geprüft. Durch die Teilnahme von klug ausgesuchten Teilnehmern der ersten Stufe und späterer Zulieferung weiterer Bibliotheken sind unendlich viele neue Titel aufgetaucht, die am Ort der Drucklegung häufig nicht bekannt waren. Es ist aber nicht zu verkennen, dass durch die Genese dieser Unternehmungen, $d$. h. die schwerpunktmäßige Verzeichnung in einigen Bibliotheken, die Druckproduktion mancher Gegenden unterrepräsentiert ist, auch wenn viele Bibliotheken inzwischen Titel gemeldet haben. ${ }^{31}$

Basis eines elektronischen Druckerkatalogs eines Ortes ist also neben dem GW für das 15. Jahrhundert ${ }^{32}$ im Anschluss das Verzeichnis der im deutschen Sprachbereich erschienenen Drucke des 16. Jahrhunderts (VD16) für Druckwerke des Erscheinungszeitraumes 1501-1600. Es umfasst derzeit mehr als 100000 Titel mit Besitznachweisen aus über 260 Bibliotheken, wird laufend aktualisiert und mit URNs und URLs für Volldigitalisate angereichert. ${ }^{33} \mathrm{Ihm}$ schließt sich das Verzeichnis der im deutschen Sprachraum erschienenen Drucke des 17. Jahrhunderts (VD17) für den Zeitraum 1601-1700 an. Das VD17 wird vom Gemeinsamen Bibliotheksverbund (GBV) als eigenständige Datenbank mit eigenem Onlinekatalog gehostet. Das VD17 enthält derzeit 299885 Ausgaben. ${ }^{34}$ Für die Titel des folgenden Jahrhunderts befindet sich das VD18 im Aufbau. ${ }^{35}$

31 Vgl. Sauer, Fässchen (wie Anm. 29), S. 147.

32 Vgl. http://www.gesamtkatalogderwiegendrucke.de.

33 Vgl. http://www.vd16.de.

34 Stand vom 17. November 2017, vgl. http://vd17.de. Leitend für Neuaufnahmen im Druckerkatalog können folglich die Richtlinien sein, wie sie z. B. für die Erfassung von VD17-Titelaufnahmen gelten: normierte Ansetzung von Personennamen nach der GND; Namen von Widmungsempfängern, Beiträgern und Zensoren nach Vorlage oder normiert; normierter Erscheinungs-/Druckort und normierte Drucker-/Verlegernamen; ausführliche Umfangs- und Illustrationsangabe; bibliografisches Format; Fingerprint; soweit möglich Gattungsbegriff nach den Richtlinien der Arbeitsgemeinschaft Alte Drucke beim GBV (AAD); vorzugsweise Volldigitalisat. 
Autopsie ist bei Verzeichnung von Beständen aus anderen Bibliotheken oder Bibliografien natürlich nicht immer möglich. Nationalbibliografie und lokaler Druckerkatalog ergänzen sich gegenseitig. Wenn schon ein älterer Zettelkatalog vorhanden ist, sollten die Titel mit dem neuen - aus den VD-Daten zusammengesetzten - Druckerkatalog verglichen und eventuell dort fehlende Titel oder Abweichungen usw. eingetragen werden. Aber die lokale Druckproduktion ist nicht nur in den VD-Bibliotheken vertreten, namentlich die lateinischen Titel können weit darüber hinaus verkauft worden sein. Daher ist die Einbeziehung ausländischer Bibliothekskataloge erforderlich wie des Universal Short Title Catalogue (USTC) ${ }^{36}$ oder von Auktions- und Antiquariatskatalogen des In- und Auslands.

Noch ein gewichtiger Partner lässt sich ausmachen, um zu wirklich tragfähigen Ergebnissen zu kommen. Unbekannte Texte sind nicht selten in Zensurakten oder bei amtlichen Aufträgen usw. in Archiven überliefert. Nicht nur die Archivbibliotheken, sondern auch die Akten selbst sind darauf durchzusehen. Gleiches gilt für die Bibliotheken in den Museen und selbständige, aber nicht in den Verbünden verzeichnete Sammlungen, z. B. Privatsammlungen. Das könnte auf Unverständnis stoßen, da eine solche Bibliothek keine Dauerhaftigkeit gewährleistet. Was für einen Verbund ein Problem ist, ist bei einer Bibliografie per Autopsie ohne Weiteres möglich. Erwünscht ist nach Absprache mit dem Besitzer die Digitalisierung, die einen solchen Titel auf Dauer der Forschung zumindest als Text zur Verfügung hält. Die archivische Überlieferung kann die Zusammenhänge der Texte klären, die Umstände der Drucklegung, Kosten usw., aber das führt über unser Thema hinaus. ${ }^{37}$

\section{Sonderproblem: Zeitungen und Periodica der Frühen Neuzeit}

Zur Aufgabe von Regionalsammlungen gehört ebenso die Sammlung von lokalen Periodica und Zeitungen vor 1800. Leider sind die erhaltenen Exemplare seit dem Beginn des Zeitungswesens im 17. Jahrhundert meistens höchst lückenhaft

35 Vgl. https://gso.gbv.de/DB=1.65/.

36 http://www.ustc.ac.uk/ für das 15. und 16. Jahrhundert. Zu erwähnen ist noch The Heritage of the Printed Book in the European Database (HPB) für ca. 1455-1830, organisiert von CERL. 37 Vgl. Eisermann, Falk: Archivgut und chronikalische Überlieferung als vernachlässigte Quelle der Frühdruckforschung. In: Gutenberg-Jahrbuch 81 (2006). S. 50-61. 
und über viele Institutionen verstreut. Das ist etwa in Köln so. ${ }^{38}$ Umso wichtiger ist es, solche Zeitungen der eigenen Region überregional aufzuspüren, zu verzeichnen und wenn möglich mit Genehmigung des Eigentümers zu digitalisieren. So ergibt sich eine digitale Rekonstruktion mit für die Regionalforschung aber nicht nur für diese - sehr interessanten Meldungen, Meinungen und Themen.

Die digitale Bereitstellung von Katalogdaten und Volltexten bietet also der Forschung viele, noch längst nicht ausgeschöpfte Möglichkeiten, die es zu nutzen gilt.

38 Vgl. Blunck, Jürgen: Die Kölner Zeitungen und Zeitschriften vor 1814. Eine Bibliographie mit Standortnachweis. Münster: Fahle 1966; Roeder, Corinna: Frühe Kölner Wochenzeitungen. Die Unternehmen der Offizinen Mertzenich und Kempen 1620 bis 1685. Köln: Greven 1998. 